JouRnal OF Universal Mathematics

Vol.1 No.2 PP.110-115 (2018)

ISSN-2618-5660

\title{
COMMON FIXED POINT THEOREMS IN $b$-MENGER SPACES
}

\author{
A. MBARKI AND R. OUBRAHIM
}

\begin{abstract}
In this work, we prove a common fixed point theorem in $b$-Menger spaces for nonlinear contractions. An example is provided to illustrate this result.
\end{abstract}

\section{INTRODUCTION}

Probabilistic metric spaces are introduced in 1942 by Menger [7]. In such spaces, the notion of distance between two points $p$ and $q$ is replaced by a distribution function $F_{p q}(x)$. Thus one thinks of the distance between points as being probabilistic with $F_{p q}(x)$ representing the probability that the distance between $p$ and $q$ is less than $x$.

Fixed point theory plays one of the important roles in nonlinear analysis. It has been applied in physical sciences, computing sciences and engineering. The first result from the fixed point theory in probabilistic metric spaces was obtained by Sehgal and Bharucha-Reid [9] in 1972 and their fixed point theorem is further generalized by many authors, for example see [4, 6, 8].

In 1989, Bakhtin [1] defined $b$-metric spaces as a generalization of metric spaces, and an extension of Banach's contraction [2] in these spaces was showed by Czerwik [3].

Recently, Mbarki et al [6] introduced a probabilistic $b$-metric spaces ( $b$-Menger spaces) as a generalization of probabilistic metric spaces (Menger spaces) and they studied topological structures and properties and showed the fixed point property for nonlinear contractions in these spaces.

In this paper, we prove the existence and uniqueness of the common fixed point for nonlinear contraction in $b$-Menger spaces.

\section{Preliminaries}

Throughout this paper, the standard notations and terminologies of probabilistic $b$-metric theory are used. For more details, we refer the reader to [6].

A nonnegative real function $f$ defined on $\mathbb{R}^{+} \cup\{\infty\}$ is called a distance distribution function (briefly, a d.d.f) if it is nondecreasing, left continuous on $(0, \infty)$, with

Date: July 09, 2018, accepted.

2000 Mathematics Subject Classification. 54E70, 54H25, 47S50.

Key words and phrases. b-Menger space, Common fixed point, Nonlinear contraction. 
$f(0)=0$ and $f(\infty)=1$. The set of all d.d.f's will be noted by $\Delta^{+}$; and the set of all $F$ in $\Delta^{+}$for which $\lim _{t \rightarrow \infty} f(t)=1$ by $D^{+}$.

A simple example of distribution function is Heavyside function in $D^{+}$

$$
H(t)= \begin{cases}0 & \text { if } \quad t \leq 0 \\ 1 & \text { if } t>0\end{cases}
$$

A commutative, associative and nondecreasing mapping $T:[0,1] \times[0,1] \rightarrow[0,1]$ is called a t-norm if and only if

(1) $T(a, 1)=a$, for all $a \in[0,1]$,

(2) $T(0,0)=0$.

As examples we mention the three typical examples of continuous t-norms as follows:

$T_{p}(a, b)=a b, T_{M}(a, b)=\operatorname{Min}(a, b)$ and $T_{L}(a, b)=\operatorname{Max}\{a+b-1,0\}$.

Definition 2.1. [6] A $b$-Menger space is a quadruple $(X, F, T, s)$ where $X$ is a nonempty set, $F$ is a function from $X \times X$ into $\Delta^{+}, T$ is a $t$-norm, $s \geq 1$ is a real number, and the following conditions are satisfied: for all $p, q, r \in X$ and $x, y>0$,

(1) $F_{p p}=H$

(2) $F_{p q}=H \Rightarrow p=q$,

(3) $F_{p q}=F_{q p}$,

(4) $F_{p q}(s(x+y)) \geq T\left(F_{p r}(x), F_{r q}(y)\right)$.

Definition 2.2. Let $(X, F)$ be a probabilistic semimetric space (i.e., (1), (2) and (3) of Definition 2.1 are satisfied). For $p$ in $X$ and $t>0$, the strong $t$-neighborhood of $p$ is the set

$$
N_{p}(t)=\left\{q \in M: F_{p q}(t)>1-t\right\} .
$$

The strong neighborhood system at $p$ is the collection $\wp_{p}=\left\{N_{p}(t): t>0\right\}$, and the strong neighborhood system for $X$ is the union $\wp=\bigcup_{p \in X} \wp_{p}$.

Let $(X, F, T, s)$ be a $b$-Menger space. The family $\Im$ consisting of $\emptyset$ and all unions of elements of the strong neighborhood system for $X$ determines a topology for $X$. Moreover, Mbarki et al [6] proved that if $T$ is continuous, $(X, F, T, s)$ endowed with this topology is a Hausdorff space and the function $F$ is in general not continuous.

Example 2.3. Let $X=\mathbb{R}$. Define $F: X \times X \rightarrow \Delta^{+}$by

$$
F_{p q}(t)=H\left(t-|p-q|^{2}\right) .
$$

By Lemma 3.1 in $[6],\left(X, F, T_{M}, 2\right)$ is a $b$-Menger space with continuous probabilistic $b$-metric $F$.

Example 2.4. [6] Let $X=\mathbb{N} \cup\{\infty\}$. Define $F: X \times X \rightarrow \Delta^{+}$as follow:

$$
F_{p q}(t)= \begin{cases}H(t) & \text { if } p=q, \\ H(t-7) & \text { if } p \text { and } q \text { are odd and } p \neq q, \\ H\left(t-\left|\frac{1}{p}-\frac{1}{q}\right|\right) & \text { if } p \text { and } q \text { are even or } p q=\infty, \\ H(t-3) & \text { otherwise. }\end{cases}
$$

It easy to show that $\left(X, F, T_{M}, 4\right)$ is a $b$-Menger space with $T_{M}$ is continuous.

Consider the sequence $x_{n}=2 n, n \in \mathbb{N}$. Then $F_{2 n \infty}(t)=H\left(t-\frac{1}{2 n}\right)$. Therefore $x_{n} \rightarrow \infty$, but $F_{2 n 1}(t)=H(t-3) \neq H(t-1)=F_{1 \infty}(t)$. Hence $F$ is not continuous at $\infty$. 
In $b$-Menger space, the convergence of sequence is defined as follows

Definition 2.5. Let $\left\{p_{n}\right\}$ be a sequence in a $b$-Menger space $(X, F, T, s)$.

(1) A sequence $\left\{p_{n}\right\}$ in $X$ is said to be convergent to $p$ in $M$ if, for every $\epsilon>0$ and $\delta \in(0,1)$, there exists a positive integer $N(\epsilon, \delta)$ such that $F_{p_{n} p}(\epsilon)>$ $1-\delta$, whenever $n \geq N(\epsilon)$.

(2) A sequence $\left\{p_{n}\right\}$ in $X$ is called a Cauchy sequence if, for every $\epsilon>0$ and $\delta \in(0,1)$, there exists a positive integer $N(\epsilon, \delta)$ such that $F_{p_{n} p_{m}}(\epsilon)>1-\delta$, whenever $n, m \geq N(\varepsilon, \delta)$.

(3) $(X, F, T, s)$ is said to be complete if every Cauchy sequence has a limit.

The letter $\Psi$ denotes the set of all function $\varphi:[0, \infty) \rightarrow[0, \infty)$ such that

$$
0<\varphi(t)<t \text { and } \lim _{n \rightarrow \infty} \varphi^{n}(t)=0 \text { for each } t>0 .
$$

Definition 2.6. [5] We say that a t-norm $T$ is of $H$-type if the family $\left\{T^{n}(t)\right\}$ is equicontinuous at $t=1$, that is,

$\forall \epsilon \in(0,1), \exists \lambda \in(0,1): t>1-\lambda \Rightarrow T^{n}(t)>1-\epsilon, \quad \forall n \geq 1$,

Where $T^{1}(x)=T(x, x), T^{n}(x)=T\left(x, T^{n-1}(x)\right)$, for every $n \geq 2$.

The t-norm $T_{M}$ is a trivial example of t-norm of $H$-type.

Lemma 2.7. [6] Let $(X, F, T, s)$ be a complete $b$-Menger space under a t-norm $T$ of $H$-type and RanF $\subset D^{+}$. Let $\left\{x_{n}\right\}$ be a sequence in $X$. If there exists a function $\varphi \in \Psi$ such that

$$
F_{x_{m+1} x_{n+1}}(\varphi(t)) \geq F_{x_{m} x_{n}}(s t) \quad(n, m \geq 0, t>0),
$$

then $\left\{x_{n}\right\}$ is a Cauchy sequence.

\section{MAIN RESUlts}

Before stating the main common fixed point theorem, we need the following concept.

Definition 3.1. Let $f$ and $g$ be two selmaps on a complete $b$-Menger space $(X, F, T, s)$. $f$ and $g$ are said to be compatible if, whenever $\left\{x_{n}\right\}$ is a sequence of point in $X$ such that $\lim _{n \rightarrow \infty} f x_{n}=\lim _{n \rightarrow \infty} g x_{n}=x$ then $f x=g x$.

Theorem 3.2. Let $(X, F, T, s)$ be a complete $b$-Menger space under a continuous t-norm $T$ of $H$-type and $R a n F \subset D^{+}$, and let $f, g: X \rightarrow X$ be maps that satisfy the following conditions:

(1) $f(X) \subseteq g(X)$,

(2) $f$ and $g$ are compatible, $g$ is continuous,

(3) There exists $\varphi \in \Psi$ such that $F_{f x f y}(\varphi(t)) \geq F_{g x g y}(s t)$, for all $x, y \in X$ and $t>0$.

Then $f$ and $g$ have a unique common fixed point in $X$.

Proof. Let $x_{0} \in X$. By condition (1), we can find $x_{1}$ such that $g\left(x_{1}\right)=f\left(x_{0}\right)$. By induction, we can define a sequence $\left\{x_{n}\right\}$ such that $g\left(x_{n}\right)=f\left(x_{n-1}\right)$. We put $y_{n}=g\left(x_{n}\right)=f\left(x_{n-1}\right)$. By condition (3), we have

$$
F_{f\left(x_{m}\right) f\left(x_{n}\right)}(\varphi(t)) \geq F_{g\left(x_{m}\right) g\left(x_{n}\right)}(s t) .
$$


So,

$$
F_{y_{m+1} y_{n+1}}(\varphi(t)) \geq F_{y_{m} y_{n}}(s t) .
$$

Then the sequence $\left\{y_{n}\right\}$ satisfies the Lemma 2.7 , hence $\left\{y_{n}\right\}$ is a Cauchy sequence. Since $X$ is complete, there is some $y \in X$ such that

$$
y_{n} \rightarrow y \quad \text { as } n \rightarrow \infty \text {. }
$$

Now we will show that $y$ is a common fixed point of $f$ and $g$. Let $\varepsilon>0$ and $\delta \in(0,1)$, since $\varphi(\varepsilon)<\varepsilon$, by the monotonicity of $F$ and the condition (3), we get

$$
\begin{aligned}
F_{y_{n+1} f(y)}(\varepsilon) & \geq F_{y_{n+1} f(y)}(\varphi(\varepsilon)) \\
& =F_{f\left(y_{n}\right) f(y)}(\varphi(\varepsilon)) \\
& \geq F_{g\left(y_{n}\right) g(y)}(s \varepsilon) .
\end{aligned}
$$

By condition (3) and since $g$ is continuous, then $f$ is also continuous. Then $\left\{y_{n}\right\}$ converges to $y$ implies that $\left\{g\left(y_{n}\right)\right\}$ converges to $g(y)$, then there exists $n_{0} \in \mathbb{N}$ such that

So,

$$
F_{g\left(y_{n}\right) g(y)}(s \varepsilon)>1-\delta \quad \text { for each } n \geq n_{0} .
$$

$$
F_{y_{n+1} f(y)}(\varepsilon)>1-\delta \quad \text { for each } n \geq n_{0}
$$

Then

$$
\lim _{n \rightarrow \infty} y_{n+1}=f(y)
$$

Since $X$ is a Hausdorff space, then

$$
f(y)=y .
$$

So by (3.1) and (3.2) we have

$$
y_{n+1}=f\left(x_{n}\right) \rightarrow y \text { and } g\left(x_{n}\right) \rightarrow y .
$$

And since $f$ and $g$ are compatible, then

$$
f(y)=g(y) .
$$

Finally,

$$
y=f(y)=g(y) .
$$

Then $y$ is a common fixed point for $f$ and $g$.

Suppose, to the contrary, that there exists another common fixed point $z$ in $X$ of $f$ and $g$. Then let $t>0$, from condition (3), since $\varphi(t)<t$ and the fact that the distance distribution functions are nondecreasing, we have

$$
\begin{aligned}
F_{y z}(\varphi(t)) & =F_{f(y) f(z)}(\varphi(t)) \\
& \geq F_{g(y) g(z)}(s t) \\
& \geq F_{g(y) g(z)}(t) \\
& =F_{y z}(t) \\
& \geq F_{y z}(\varphi(t)) .
\end{aligned}
$$

Thus

$$
F_{y z}(\varphi(t))=F_{y z}(t) .
$$

By the same arguments, we get

$$
F_{y z}\left(\varphi^{n}(t)\right)=F_{y z}(t), \quad \text { for all } n \geq 1 .
$$


Suppose that there exists $\alpha>0$ such that $F_{y z}(\alpha)<1$. Since $F_{y z} \in D^{+}$, then $\lim _{t \rightarrow \infty} F_{y z}(t)=1$, so there exists $\beta>\alpha$ such that

$$
F_{y z}(\beta)>F_{y z}(\alpha) .
$$

There exists a positive integer $n>1$ such that $\varphi^{n}(\beta)<\alpha$ because $\lim _{n \rightarrow \infty} \varphi^{n}(\beta)=0$. Then, the fact that $F_{y z}$ is nondecreasing function, we get

$$
F_{y z}\left(\varphi^{n}(\beta)\right) \leq F_{y z}(\alpha) .
$$

Hence

$$
F_{y z}(\beta)=F_{y z}\left(\varphi^{n}(\beta)\right) \leq F_{y z}(\alpha),
$$

a contradiction. Therefore $F_{y z}(t)=1$ for all $t>0$, since $F_{y z} \in D^{+}$. Hence $F_{y z}=H$.

Then $y=z$.

Example 3.3. Let $X=[0, \infty)$. Define $F: X \times X \rightarrow \Delta^{+}$as follows

$$
F_{x y}(t)=H\left(t-|x-y|^{2}\right) .
$$

By Examples 3.2, 3.3 and Lemma 3.1 in $[6],\left(X, F, T_{M}, 2\right)$ is a complete $b$-Menger and is not a standard Menger space.

Define $f(x)=\frac{x}{12}$ and $g(x)=\frac{x}{4}$ in $X$. It is evident that $f(X) \subseteq g(X)$. Also, for $\varphi(t)=\frac{16 t}{77}$,

$$
\begin{aligned}
F_{f(x) f(y)}\left(\frac{16 t}{77}\right) & =H\left(\frac{16 t}{77}-\left|\frac{x}{12}-\frac{y}{12}\right|^{2}\right) \\
& =H\left(\frac{\frac{16 t}{77}}{\frac{1}{144}}-|x-y|^{2}\right) \\
& =H\left(\frac{2 t}{\frac{1}{16}}-|x-y|^{2}\right) \\
& =H\left(2 t-\left|\frac{x}{4}-\frac{y}{4}\right|^{2}\right) \\
& =F_{g(x) g(y)}(2 t) .
\end{aligned}
$$

Thus all the conditions of Theorem 3.2 are satisfied and $f$ and $g$ have the common fixed point 0.

\section{REFERENCES}

[1] Bakhtin I.A., Contracting mapping principle in an almost metric space. (Russian) Funkts. Anal. 30, 26-37 (1989).

[2] Banach S., Sur les opérations dans les ensembles abstraits et leur applications aux équations intégrales. Fundam. Maths. 3, 133-181 (1922).

[3] Czerwik S., Nonlinear set-valued contraction mappings in b-metric spaces, Att Sem Math Univ. Modena 46(2), 263-276, (1998).

[4] Elamrani M., Mbarki A. and Mehdaoui B., Nonlinear contarctions and semigroups in general complete probabilistic metric spaces, Panam. Math. J. 11, 4, pp. 79-87, (2001).

[5] Hadzić O., A fixed point theorem in Menger spaces, Publ. Inst. Math. (Beograd) T 20, 107$112,(1979)$.

[6] Mbarki A. and Oubrahim R., Probabilistic b-metric spaces and nonlinear contractions. Fixed Point Theory and Applications (2017) 2017: 29.

[7] Menger K., Statistical metrics, Proc. Natl. Acad. Sci. 28, 535-537, (1942).

[8] Schweizer B. and Sklar A., Probabilistic Metric Spaces, North-Holland Series in Probability and Applied Mathimatics, 5, (1983). 
[9] Sehgal V. M. and Bharucha-Reid A. T., Fixed point theorems of contractions mappings in probabilistic metric spaces, Math. Systemes Theory, 6, 97-102, (1972).

(A. Mbarki) Ano Laboratory, National School of Applied Sciences, P.O. Box 669, Oujda University, Morocco.

E-mail address: dr.mbarki@gmail.com

(R. Oubrahim) ANO Laboratory, Faculty of Sciences, Oujda University, 60000 Oujda, Morocco.

E-mail address: rchd.oubrahim@gmail.com 